\title{
An overview of Lassa fever
}

\author{
Abdulmutalab Musa 123 *
}

\begin{abstract}
Lassa fever is a viral hemorrhagic fever caused by Lassa virus (Lassa mammarenavirus), a negative-sense singlestranded RNA virus of the Arenaviridae family. ${ }^{[1][2] ~ I n ~ m o s t ~ c a s e s ~ L a s s a ~ v i r u s ~ i n f e c t i o n ~ i s ~ a s y m p t o m a t i c ~(p r e s e n t i n g ~}$ no symptom). ${ }^{[1]}$ When symptomatic it is characterized by mild acute febrile disease to a chronic fatal disease with severe toxaemia, capillary leak, hemorrhagic situations, shock and multiple organ failure. ${ }^{[3]}$ Early diagnosis of Lassa fever is very important because of the transmissibility of infection, the need for potent isolation of infected persons and for containing potentially infectious specimens during laboratory testing. [4][5]

Lassa fever was first elucidated in the 1950s, but the virus was not recognized until 1969 when it infected two missionary nurses in Lassa Village, Borno State, Northeastern Nigeria. ${ }^{[1]}$ Natal multimammate rat or common African rat of Mastomys genus are the reservoir of Lassa virus. ${ }^{[1]}$ When the rodents become infected with Lassa virus, they infect humans through their urine and faeces, but remain unharmed. ${ }^{6]}$

Because of its similarities with other febrile diseases such as malaria, typhoid, Ebola hemorrhagic fever, early detection is difficult. Thus when persons have persistent fever not responding to normal conventional therapies, they should be screened for other possible causes (especially in endemic regions). When the presence of Lassa fever is established in a community, immediate isolation of infected individuals, screening, standard infection prevention and control practices and meticulous contact tracing can halt outbreaks. ${ }^{[1]}$ Treatment involves supportive measures and early use of the antiviral drug ribavirin.
\end{abstract}

Note: This article has been updated since its initial publication on 15 Jun 2019 (summary of changes). The previous version is archived at this link as a record.

\section{Pathophysiology}

\section{Main article: Lassa virus}

Lassa virus is a single-stranded, negative-sense RNA virus (Figure 1). ${ }^{[4]}$ The transmission of Lassa virus to humans can occur through direct contact and aerosols generated from the urine or feces of an infected rodent. ${ }^{[6]}$ Natal multimammate rats shed the virus in urine and droppings, direct contact with these excreta, through touching soiled objects, eating contaminated food, or exposure to open cuts or sores, can lead to infection. ${ }^{[6][7]}$ There have been reports of sexual transmission of Lassa fever but it is rare. ${ }^{[8]}$ High serum virus titres, combined with disseminated replication in tissues and absence of neutralizing antibodies (immuno-compromisation), lead to the development of Lassa fever. ${ }^{[4]}$

${ }^{1}$ Sir Muhammad Sanusi Specialist Hospital, Kano

2 Ladoke Akintola University of Technology, Ogbomoso, Oyo State, Nigeria

3 Islamic Medical Association of Nigeria

*Author correspondence: laamiido@gmail.com

ORCID: 0000-0002-9319-198X

Licensed under: CC-BY

Received 25-09-2018; accepted 15-06-2019; updated 15-10-2019
However, an intact and active immune response is protective against developing symptoms by mounting the early innate immune response in order to prevent further infection and virus growth, which in turn attenuates humoral and cell-mediated immunity. ${ }^{[6][9][10]}$ Due

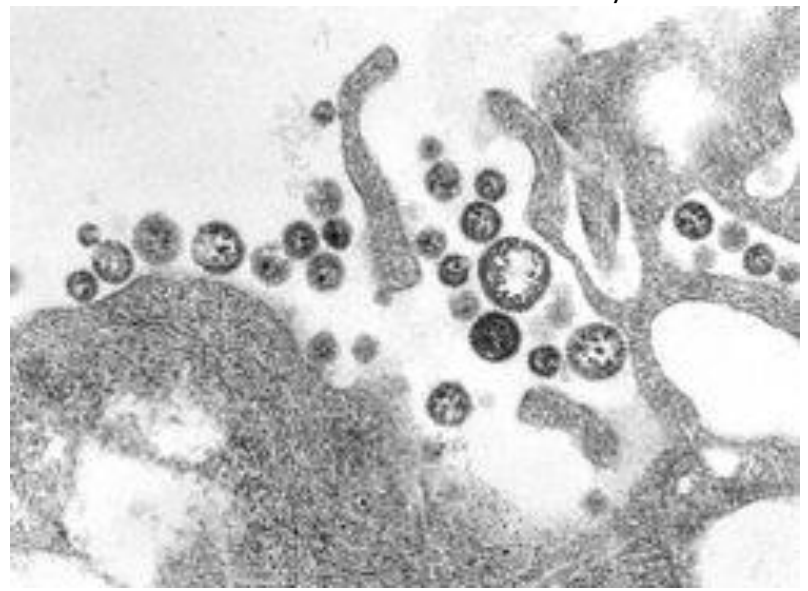

Figure 1| A transmission electron micrograph (TEM) of a number of Lassa virus virions adjacent to some cell debris. The virus, a member of the virus family Arenaviridae, causes Lassa fever.

Centers for Disease Control and Prevention, public domain 
to limited data on Lassa fever, the immune responses against it and its pathogenesis are poorly understood. ${ }^{[6]}$ As such, it is not well understood how viral infection leads to sepsis-like symptoms, cytokine storms or bacterial co-infection. ${ }^{[11][12]}$

There are several pathways involved in the pathogenesis of Lassa fever. ${ }^{[4][13]}$ Similar to the pathogenesis of sepsis, induction of uncontrolled cytokine expression can be triggered by Lassa virus infection. ${ }^{[4]}$ Systemic viral-induced immunosuppression can also be implicated in severe Lassa virus infections. ${ }^{[4]}$

Two immunoglobulins (lgM and IgG antibody isotypes) are produced in Lassa virus infected persons, because both can be

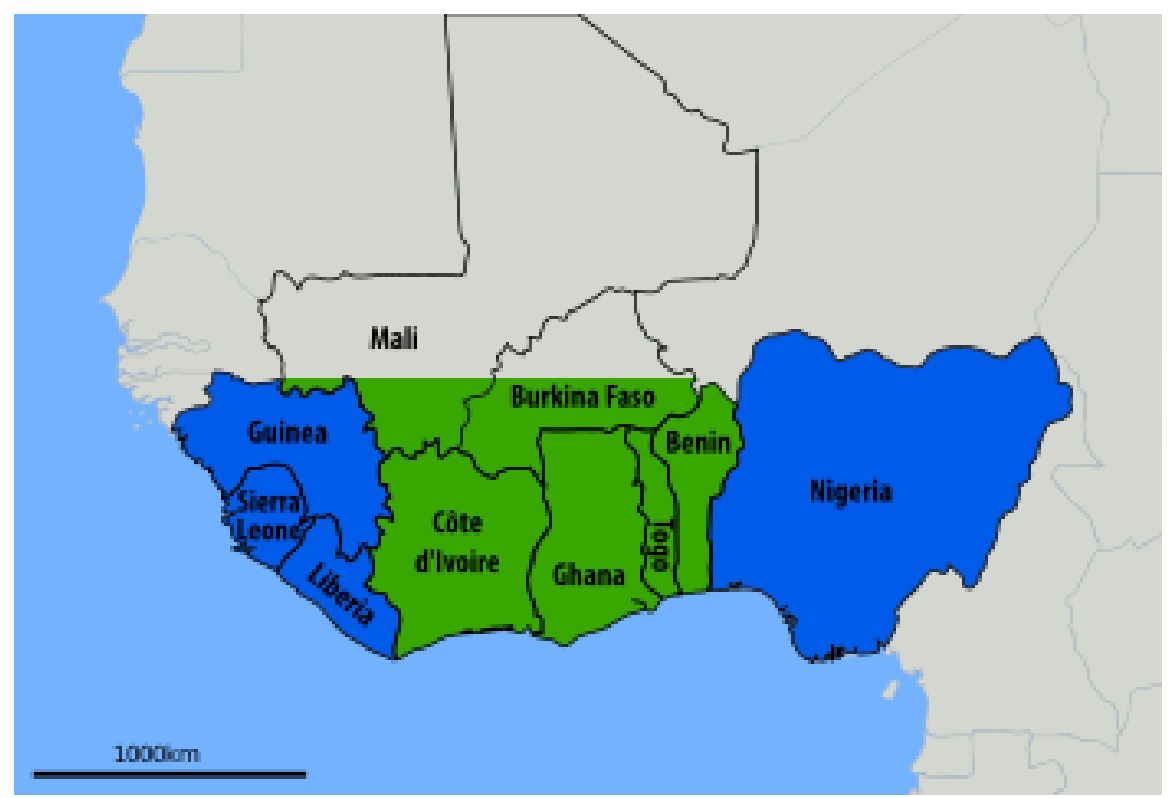

Figure 2 | Lassa Fever Outbreak Distribution Map. Countries reporting endemic disease and substantial outbreaks of Lassa Fever in blue. Countries reporting few cases, periodic isolation of virus, or serological evidence of infection in green. Countries with unknown status in grey. ${ }^{[18]}$

Centers for Disease Control and Prevention, public domain present in viremic persons, and possibly only non-neutralizing antibodies are produced early in the infectious process, this makes the antibodies to remain present in many people across West Africa. ${ }^{[4][13]}$

While late antibodies are protective because they neutralize the virus, Early antibodies are not neutralizing making them resistant; ${ }^{[4][13]}$ this is because proteinous surface of the Lassa virus is protected by under-processed glycans form with structurally distinct clusters. $^{[6][14][15]}$

The main underlying feature of Lassa fever is that the vascular bed is attacked by the virus, with resultant micro-vascular damage and changes in vascular permeability. ${ }^{[4][16]}$ Secondary resultant of capillary leak syndrome and reduced blood volume may include increased cardiac activity, local tissue acidosis, anoxia and reduction in blood circulation, thus leading to the shock syndrome. ${ }^{[16]}$ Generally it is clear that liver damage occurs in almost all cases of Lassa fever in different levels. ${ }^{[4]}$

Pre-renal acute kidney failure, lactic acidaemia, hyperkalaemia and reduced perfusion and oxygenation of vital tissues follows and progress to fatal outcome. ${ }^{[16][17]}$ The secondary effects of micro-vascular damage include alterations in pulmonary function due to several mechanisms. ${ }^{[17]}$

\section{Frequency (epidemiology)}

Estimating the true incidence and mortality of Lassa Fever is extremely difficult due to the non-specific clinical presentation; poor surveillance systems; sizeable human migration, uneasy landscape and lack of standard laboratory confirmation. ${ }^{[19]}$

Nevertheless, Lassa virus frequently infects people in West Africa (Figure 2) with approximately $80 \%$ being asymptomatic. [20] Studies show up to 300,000 500,000 cases and about 5,000 deaths annually. Lassa fever is endemic in some parts of West Africa, which include Sierra Leone, Liberia, Ghana, Guinea and Nigeria. ${ }^{[20]}$

There have been reports of Lassa fever in neighboring countries; In 2016, two cases were reported in Togo, [21] and 6 confirmed cases in Benin. ${ }^{[22]}$ In the US on 25 May 2015 , there was a confirmed case in a US returnee from Liberia. ${ }^{[23]}$ There have also been reports of imported cases of Lassa fever in European countries including Sweden, ${ }^{[24]}$ Germany, $^{[25]}$ The Netherlands ${ }^{[26]}$ and the United Kingdom. ${ }^{[27]}$

$\mathbf{2}$ of $\mathbf{7} \mid$ WikiJournal of Medicine 


\section{Outbreak in Nigeria}

In Nigeria, from 1 January to 20 May 2018, 1940 suspected cases have been reported from 21 states. ${ }^{[28]}$ Of these, 431 were confirmed positive, 10 are probable, 1495 negative. ${ }^{[28][1]}$ A total of 6489 contacts have been identified in 20 states since January 2019 to March 2019, a total of 2034 suspected cases have been reported from 21 states. ${ }^{[28]}$ Of these, 526 were confirmed positive, 15 were probable and 1693 negative (not a case). ${ }^{[28]}$ Out of these, 17 health care workers have been affected in six states, with four deaths (case fatality rate $=29 \%)^{[28]}$

\section{Presentation}

Lassa fever has an incubation period of $6-21$ days. ${ }^{[1]}$ The onset of lassa fever is usually asymptomatic and when symptomatic it is usually subtle, starting with fever and malaise. When it progresses, it presents with sore throat, headache, achy muscle, nausea, vomiting, chest pain, diarrhea, cough, and abdominal pain. ${ }^{[1][29]}$ In critical cases systemic involvement occurs with the following:

- Respiratory: pleural effusion, epistaxis, rales, rhonchi, stridor, cough, wheezing, pharyngitis, and dyspnoea ${ }^{[17][30]}$

- Gastrointestinal: hematemesis, melena, gingival bleeding, dysphagia, hepatitis, and hepatic tenderness $^{[17][30]}$

- Renal: hematuria, dysuria ${ }^{[17]}$

- Cardiovascular: pericarditis, hypotension, and tachycardia[17][30]

- Nervous: encephalitis, cloudy sensorium, seizures, disorientation, coma, unilateral or bilateral hearing deficit $^{[17][30]}$

- Vascular: petechial and ecchymotic cuteneous lesions, facial and cervical edema ${ }^{[17][30]}$

Temporary hair loss and gait interference may occur during recovery. ${ }^{[1][31]}$

Lassa fever is usually fatal within 14 days of inception. ${ }^{[1][32]}$

\section{Causes and transmission}

Lassa virus is zoonotic, ${ }^{[1][33]}$ as it spreads specifically from Natal multimammate mice (Mastomys natalensis). ${ }^{[1][33]}$ The mice are the most abundant rodents in equatorial Africa, omnipresent in households and consumed as food in some areas. ${ }^{[34]}$ Infection occurs by exposure to rat excreta directly or indirectly via contaminated foodstuffs. ${ }^{[33]}$ Infection can also occur by inhalation of tiny particles (aerosols) of infectious materials, but airborne transmission is unlikely as there is no evidence to support that. ${ }^{[1]}$ It is possible to acquire infection through broken skin or mucous membranes that is directly exposed to infectious materials, and through rat bites. ${ }^{[1][33]}$ In addition, the virus can also be contracted via contaminated hospital equipment, such as re-used needles and improper sterilization. ${ }^{[1]}$ The presence of Lassa virus in the semen indicates high risk of sexual transmission but viral load is not enough to cause infection. ${ }^{[35]}$

\section{Diagnosis}

Clinical diagnosis of Lassa fever is usually difficult, this is as a result of its vague symptoms. ${ }^{[1]}$ Lassa fever is hard to differentiate from other febrile diseases such as malaria, typhoid, influenza, relapsing fever, leptospirosis and other hemorrhagic fevers e.g. yellow fever, dengue, Marburg and Ebola. ${ }^{[1][36]}$

The following laboratory tests can be conducted: ${ }^{[1]}$

- Enzyme-linked immunosorbent assays (ELISAs) can be used to detect specific immunoglobulin G (lgG) or viral antigens in acute serum samples from persons with Lassa fever (it can be detected even in acute phase). ${ }^{[37]}$

- Reverse transcription polymerase chain reaction (RT-PCR) assay can be used in early stage to detect the virus using inactivated virus. it is very helpful in areas where Biosafety Level 4 (BSL4) laboratories cannot be found especially in west Africa. ${ }^{[38]}$

- Virus cultivation and identification technique (virus isolation by cell culture). However, this requires 3 10 days or longer for definitive identification. ${ }^{\text {[39][40] }}$

- Blood cultures to differentiate from other pathogens (e.g. typhoid) ${ }^{[41]}$ and blood smear to differentiate from malaria parasite ${ }^{[42]}$ as the virus can present concomitantly with other diseases. ${ }^{\text {[2] }}$

- General biochemical tests such as full blood count, erythrocyte sedimentation rate; hematocrit volume (to exclude anemia); white blood cell count (to exclude lymphopenia); platelet count (to exclude thrombocytopenia), coagulation studies (to exclude coagulopathies) and liver and kidney function tests (serum liver enzymes have been found to be positive clinical markers). ${ }^{[43]}$

The WHO guidelines for the collection, storage, and handling of Ebola virus specimens testing can be ad- 
hered to when testing for Lassa virus. ${ }^{[44]}$ Thorough adherence to biosafety level 4 (BSL-4) precautions is pertinent when handling suspected specimens. ${ }^{[4]}$ However, BSL-4 laboratories are limited worldwide, when not available, samples should be handled in biosafety level 2 or 3 cabinets or preferably they should be inactivated so as to be handled under BSL-2 precautions. $^{[44][45]}$ In West Africa false-negative results were reported due to the probe or antibody pairs not adequately binding to the target, This could be due to the high diversity of nucleotide and amino acid of the Lassa virus isolates sequenced. ${ }^{[44]}$ For instance, a widely used RT-PCR assay in West Africa ${ }^{[44]}$ was modified when when primer-template mismatch was found to give false negatives results. ${ }^{[44]}$

Currently, two national laboratories in Nigeria are supporting the laboratory confirmation PCR tests. ${ }^{[46]}$ All the samples are also tested for Ebola, dengue and yellow fever (which have so far tested negative). ${ }^{[46]}$

\section{Treatment}

\section{Main article: Ribavirin}

Supportive (symptomatic) management includes bed rest; close observation and monitoring; serial laboratory tests; analgesics (e.g. Ibuprofen); tepid sponging and antipyretic drugs (e.g. acetaminophen) to reduce fever; antiemetic drugs (e.g. metoclopramide and promethazine); prompt fluid and electrolyte replacement; diuretics (e.g. furosemide) for fluid retention; oxygen therapy; blood and or platelet transfusion; and management of other complications. ${ }^{[47]}$

In terms of specific management, early initiation of ribavirin is most effective treatment. ${ }^{[48]}$ Intravenous interferon may also be given alongside ribavirin. ${ }^{[49]}$

\section{Ribavirin}

The generic drug ribavirin is a synthetic broad-spectrum antiviral nucleoside (guanosine). ${ }^{[50]}$ Its international brand names include Copegus, Ibavyr, Moderiba, Virazole, Virazide, Rebetol, Ribasphere, RibaTab and Riboflax, among many others. ${ }^{[50]}$

\section{Medical use}

Ribavirin is the primary drug of choice in treating Lassa fever infection. ${ }^{[50]}$ It has also shown effectiveness in treating other viral infections like hepatitis B. ${ }^{[50][51]}$

\section{Contraindications}

Documented or known hypersensitivity, compromised renal function, or renal failure (creatinine clearance $<30$ $\mathrm{ml} / \mathrm{min}$ ), pregnancy, hemoglobinopathies (e.g. thelassemia major, sickle cell anemia with hemoglobin level less than $8 \mathrm{~g} / \mathrm{dl}$, etc.) are (relative) contraindications to ribavirin. ${ }^{[52]}$

\section{Adverse effects}

- Hemolytic anemia may occur within $1-2$ weeks of initiating therapy. It is recommended that pack cell volume be obtained before treatment is initiated and re-obtained at week 2 and week 4 of therapy or as clinically indicated. ${ }^{[52]}$

- Fatal and non-fatal myocardial infarction can occur in persons with ribavirin-induced anemia. Cardiac assessment should be done before commencement of therapy. Individuals with known cardiac compromise will require electrocardiography monitored during therapy. ${ }^{[52]}$

- Hypersensitivity, e.g. uticaria, angioedema, bronchoconstriction and anaphylaxis ${ }^{[52]}$

- Bone marrow suppression (pancytopenia) ${ }^{[52]}$

- Unusual tiredness and weakness ${ }^{[52]}$

- Insomnia, depression, irritability and suicidal behavior have been reported with oral administration ${ }^{[52]}$

- Ocular problems ${ }^{[52]}$

- Mild hepatic and renal impairment ${ }^{[52]}$

\section{Drug interactions}

Ribavirin inhibits the phosphorylation of zidovudine and ostavudin. ${ }^{[53]}$

\section{Pharmacodynamics}

Although the mechanism of ribavirin remains unclear, ribavirin appears to be a non-specific antiviral agent with most of its efficacy due to incorporation of ribavirin into the viral genome. ${ }^{[49]}$ When cells are exposed to ribavirin, there is reduction in intracellular guanosine triphosphate (a requirement for translation, transcription and replication in viruses). ${ }^{[49]}$ Therefore ribavirin effectively inhibits viral replication and translation by inhibiting DNA and RNA synthesis. ${ }^{[49]}$

\section{Ribavirin in pregnancy}

Ribavirin can cause birth defects and or death of exposed fetuses. ${ }^{[52]}$ Studies done on animal species, reveals that it had considerable teratogenic and or embryocidal effects. ${ }^{[52]}$ These adverse effects occurred at

\begin{tabular}{l|l}
4 of 7 & WikiJournal of Medicine
\end{tabular} 
even lower than recommended human dose of ribavirin. ${ }^{[52]}$

Ribavirin therapy should not be commenced in females until serum pregnancy test is negative. ${ }^{[53]}$ Care should be taken to prevent pregnancy in females with male infected partners (as it can be secreted via sperm). ${ }^{[53]}$ To prevent pregnancy the female partners should be instructed to use 2 contraceptive (condoms and any other non barrier method) for 6 months after their partners have been weaned off treatment. ${ }^{[53][54]}$

Note: In pregnancy the goal is to save the mother's life. ${ }^{[47]}$ As ribavirin therapy cannot be started with pregnancy because of the risks it poses to the mother and fetus. ${ }^{[47]}$ Conservative management can be explored in pregnant women infected with Lassa fever, but in some cases labor must be induced to save the mother's life after which ribavirin therapy can begin immediately, especially in third trimesters. ${ }^{[4]]}$

\section{Post Exposure Prophylaxis (PEP)}

Individuals who come in contact with infected persons or equipment (i.e. via broken skin, mucous membrane or needle stick injuries) approximately within 2 days of exposure, are given $800 \mathrm{mg}$ of ribavirin daily or $400 \mathrm{mg}$ twice daily for 10 days. ${ }^{[55]}$ This was the proposal of Vito et al. in 2010, following their experimental research in Sierra Leone's Lassa ward on only 25 people who were exposed to the virus, all being negative after the prophylaxis. ${ }^{[55]}$ But there is no substantial evidence to support the effectiveness of immediate initiation of PEP. ${ }^{[55]}$

However the CDC recommends placing high-risk exposed individuals under medical surveillance for 21 days and treating presumptively with ribavirin if clinical evidence of viral hemorrhagic fever develops. ${ }^{[56]}$

\section{Prognosis}

Mortality rate during epidemics can be as high as 50\%, ${ }^{[1]}$ About $15-20 \%$ of those hospitalized with Lassa fever die from the illness. ${ }^{[57]}$ The mortality rate of pregnant women infected with Lassa fever is $80 \%, 87 \%$ at second and third trimester respectively and $95 \%$ experience fetal deaths.

The occurrence of deafness is $25 \%$ in persons cured from the disease. half of these persons regain hearing partially after $1-3$ months. $^{[1]}$

$\mathbf{5}$ of $\mathbf{7} \mid$ WikiJournal of Medicine

\section{Prevention and control}

\section{Main article: Prevention of viral hemorrhagic fever}

Initiating good "community hygiene" which will prevent rodents from entering homes. ${ }^{[1]}$ Other steps include storing foodstuffs in rodent-proof containers, good sewage and garbage disposal and keeping ratpredator such as cats. ${ }^{[1]}$ Rodents are abundant in endemic regions and very hard to completely eliminate, so it is advised that contact should be prevented as much as possible. ${ }^{[1]}$ While caring for sick persons, caregivers should prevent contact with all bodily fluid. The government and stakeholder should also ensure safe burial process are sustained. ${ }^{[1]}$

Clinical staffs managing persons infected or suspected to have the disease should maintain standard infection prevention and control protocols when attending to these individuals, despite their postulated diagnosis. ${ }^{[58][30]}$

Proper isolation of suspected and confirmed cases of Lassa fever, good quarantine protocols, health education and rigorous contact tracing should be employed by the government and health care agencies. ${ }^{[59]}$ Drugs, equipment and appropriate expertise should also be readily available to control the spread in time. ${ }^{[59]}$

\section{Additional information}

\section{Competing interests}

No competing interest.

\section{References}

1. "Lassa fever". www. who.int. Retrieved 2019-05-25.

2. Oti, Victor B. (2018-11-05). "A Reemerging Lassa Virus: Aspects of Its Structure, Replication, Pathogenicity and Diagnosis" (in en). Current Topics in Tropical Emerging Diseases and Travel Medicine. doi:10.5772/intechopen.79072.

3. Monath, T. P.; Casals, J. (1975). "Diagnosis of Lassa fever and the isolation and management of patients". Bulletin of the World Health Organization 52 (4-6): 707-715. ISSN 0042-9686. PMID 1085225. PMC PMC2366641.

4. Yun, Nadezhda E.; Walker, David H. (2012-10-09). "Pathogenesis of Lassa Fever". Viruses 4 (10): 2031-2048. doi:10.3390/v4102031. ISSN 1999-4915. PMID 23202452. PMC PMC3497040.

5. Monath, T. P.; Casals, J. (1975). "Diagnosis of Lassa fever and the isolation and management of patients". Bulletin of the World Health Organization 52 (4-6): 707-715. ISSN 0042-9686. PMID 1085225. PMC PMC2366641.

6. Hallam, Hoai J.; Hallam, Steven; Rodriguez, Sergio E.; Barrett, Alan D. T.; Beasley, David W. C.; Chua, Arlene; Ksiazek, Thomas G.; Milligan, Gregg N. et al. (2018-03-20). "Baseline mapping of Lassa 
fever virology, epidemiology and vaccine research and development". NPJ Vaccines 3. doi:10.1038/s41541-018-0049-5. ISSN 2059-0105. PMID 29581897. PMC 5861057.

7. "Transmission of Lassa Fever". www.cdc.gov. 2019-03-06. Retrieved 2019-04-09.

8. Oshin, Babafemi A. (2019-04-09) (in en). Rat eating, sexual transmission and the burden of Lassa fever disease.

9. Zapata, Juan Carlos; Medina-Moreno, Sandra; Guzmán-Cardozo, Camila; Salvato, Maria S. (2018-10-28). "Improving the Breadth of the Host's Immune Response to Lassa Virus". Pathogens 7 (4). doi:10.3390/pathogens7040084. ISSN 2076-0817. PMID 30373278. PMC 6313495.

10. Brosh-Nissimov, Tal (2016-04-30). "Lassa fever: another threat from West Africa". Disaster and Military Medicine 2. doi:10.1186/s40696-016-0018-3. ISSN 2054-314X. PMID 28265442. PMC 5330145.

11. Lin, Gu-Lung; McGinley, Joseph P.; Drysdale, Simon B.; Pollard, Andrew J. (2018-09-27). "Epidemiology and Immune Pathogenesis of Viral Sepsis". Frontiers in Immunology 9. doi:10.3389/fimmu.2018.02147. ISSN 1664-3224. PMID 30319615. PMC 6170629.

12. USA US9193705B2, Cunningham J; Lee K \& Ren T et al., "Small molecule inhibitors of ebola and lassa fever viruses and methods of use"

13. "Lassa". Viral Hemorrhagic Fever Consortium. Retrieved 2019-0409.

14. Crispin, Max; Bowden, Thomas A.; Strecker, Thomas; Huiskonen, Juha T.; Moser, Felipe; Li, Sai; Seabright, Gemma E.; Allen, Joel D. et al. (2018-07-10). "Structure of the Lassa virus glycan shield provides a model for immunological resistance" (in en). Proceedings of the National Academy of Sciences 115 (28): 73207325. doi:10.1073/pnas.1803990115. ISSN 0027-8424. PMID 29941589.

15. Robinson, James E.; Hastie, Kathryn M.; Cross, Robert W.; Yenni, Rachael E.; Elliott, Deborah H.; Rouelle, Julie A.; Kannadka, Chandrika B.; Smira, Ashley A. et al. (2016-05-10). "Most neutralizing human monoclonal antibodies target novel epitopes requiring both Lassa virus glycoprotein subunits". Nature Communications 7. doi:10.1038/ncomms11544. ISSN 2041-1723. PMID 27161536. PMC 4866400.

16. Brisse, Morgan E.; Ly, Hinh (2019-03-13). "Hemorrhagic FeverCausing Arenaviruses: Lethal Pathogens and Potent Immune Suppressors". Frontiers in Immunology 10. doi:10.3389/fimmu.2019.00372. ISSN 1664-3224. PMID 30918506. PMC 6424867.

17. Monath, T. P.; Casals, J. (1975). "Diagnosis of Lassa fever and the isolation and management of patients". Bulletin of the World Health Organization 52 (4-6): 707-715. ISSN 0042-9686. PMID 1085225. PMC PMC2366641.

18. "Outbreak Distribution Map of Lassa Fever". www.cdc.gov. CDC. 2019-03-04. Retrieved 2019-04-27.

19. Grant, Donald S.; Khan, Humarr; Schieffelin, John; Bausch, Daniel G. (2014). Emerging Infectious Diseases. Elsevier. pp. 37-59. doi:10.1016/b978-0-12-416975-3.00004-2. ISBN 9780124169753.

20. Behrens, Ron; Houlihan, Catherine (2017-07-12). "Lassa fever" (in en). BMJ 358: j2986. doi:10.1136/bmj.j2986. ISSN 1756-1833. PMID 28701331.

21. "Lassa Fever-Togo". WHO. Retrieved 2019-01-11.

22. "Lassa Fever-Benin". WHO. Retrieved 2019-01-11.

23. "Lassa Fever - United States of America". WHO. Retrieved 201901-11.

24. "Lassa fever-Sweden". WHO. Retrieved 2019-01-11.

25. "Lassa Fever-Germany". WHO. Retrieved 2019-01-11.

26. "2000 - Imported case of Lassa fever in The Netherlands Update". WHO. Retrieved 2019-01-11.
27. "Imported case of Lassa fever in United Kingdom". WHO. Retrieved 2019-01-11.

28. "Nigeria Centre for Disease Control". www.ncdc.gov.ng. Retrieved 2019-04-09.

29. "Lassa fever - Symptoms, diagnosis and treatment". bestpractice.bmj.com. BMJ. Retrieved 2019-06-22.

30. Ogbu, O.; Ajuluchukwu, E.; Uneke, C. J. (2007-3). "Lassa fever in West African sub-region: an overview". Journal of Vector Borne Diseases 44(1): 1-11. ISSN 0972-9062. PMID 17378212.

31. Behrens, Ron; Houlihan, Catherine (2017-07-12). "Lassa fever" (in en). BMJ 358: j2986. doi:10.1136/bmj.j2986. ISSN 0959-8138. PMID 28701331

32. Greenky, David; Knust, Barbara; Dziuban, Eric J. (2018-05-01). "What Pediatricians Should Know About Lassa Virus". JAMA $\begin{array}{llll}\text { pediatrics } & 172 & \text { (5): } & \text { 407-408. }\end{array}$ doi:10.1001/jamapediatrics.2017.5223. ISSN 2168-6203. PMID 29507948. PMC PMC5970952.

33. Kafetzopoulou, L. E.; Pullan, S. T.; Lemey, P.; Suchard, M. A.; Ehichioya, D. U.; Pahlmann, M.; Thielebein, A.; Hinzmann, J. et al. (2019-01-04). "Metagenomic sequencing at the epicenter of the Nigeria 2018 Lassa fever outbreak"(in en). Science 363 (6422): 7477. doi:10.1126/science.aau9343. ISSN 0036-8075.

34. Hussainia, Nafiu; Abdulhamid, Abdurrahman (2018-01-01). "Effects of quarantine on transmission dynamics of Lassa fever". Bayero Journal of Pure and Applied Sciences11 (1): 397-407-407. ISSN 2006-6996.

35. Oshin, Babafemi A. (2019-04-27) (in en). Rat eating, sexual transmission and the burden of Lassa fever disease.

36. "Lassa fever". www. who.int. Retrieved 2019-06-20.

37. Bausch, D. G. Rollin, P. E. Demby, A. H. Coulibaly, M. Kanu, J. Conteh, A. S. Wagoner, K. D. McMullan, L. K. Bowen, M. D. Peters, C. J. Ksiazek, T. G. Diagnosis and Clinical Virology of Lassa Fever as Evaluated by Enzyme-Linked Immunosorbent Assay, Indirect Fluorescent-Antibody Test, and Virus Isolation. American Society for Microbiology. OCLC 679252357.

38. Koehler, Jeffrey; Raabe, Vanessa (2017-06-01). "Laboratory Diagnosis of Lassa Fever" (in en). Journal of Clinical Microbiology 55 (6): 1629-1637. doi:10.1128/JCM.00170-17. ISSN 0095-1137. PMID 28404674.

39. Raabe, Vanessa; Koehler, Jeffrey (2017-6). Kraft, Colleen Suzanne. ed. "Laboratory Diagnosis of Lassa Fever" (in en). Journal of Clinical Microbiology 55 (6): 1629-1637. doi:10.1128/JCM.00170-17. ISSN 0095-1137. PMID 28404674. PMC PMC5442519.

40. Panning, Marcus; Emmerich, Petra; Ölschläger, Stephan; Bojenko, Sergiusz; Koivogui, Lamine; Marx, Arthur; Lugala, Peter Clement; Günther, Stephan et al. (2010-6). "Laboratory Diagnosis of Lassa Fever, Liberia". Emerging Infectious Diseases 16(6): 10411043. doi:10.3201/eid1606.100040. ISSN 1080-6040. PMID 20507774. PMC PMC3086251.

41. Kumar, Praveen; Kumar, Ruchika (2017-3). "Enteric Fever" (in en). The Indian Journal of Pediatrics 84 (3): 227-230. doi:10.1007/s12098-016-2246-4. ISSN 0019-5456.

42. Kattenberg, Johanna H; Ochodo, Eleanor A; Boer, Kimberly R; Schallig, Henk DFH; Mens, Petra F; Leeflang, Mariska MG (201112). "Systematic review and meta-analysis: rapid diagnostic tests versus placental histology, microscopy and PCR for malaria in pregnant women" (in en). Malaria Journal 10 (1). doi:10.1186/1475-2875-10-321. ISSN 1475-2875.

43. Salvato, Maria S.; Lukashevich, Igor S.; Medina-Moreno, Sandra; Zapata, Juan Carlos (2018). "Diagnostics for Lassa Fever: Detecting Host Antibody Responses". Methods in Molecular Biology (Clifton, N.J.) 1604: 79-88. doi:10.1007/978-1-4939-6981-4_5. ISSN 19406029. PMID 28986826.

44. Raabe, Vanessa; Koehler, Jeffrey (06 2017). "Laboratory Diagnosis of Lassa Fever". Journal of Clinical Microbiology 55 (6): 1629-

$\mathbf{6}$ of $\mathbf{7} \mid$ WikiJournal of Medicine 
1637. doi:10.1128/JCM.00170-17. ISSN 1098-660X. PMID 28404674. PMC 5442519.

45. Asogun, Danny A.; Adomeh, Donatus I.; Ehimuan, Jacqueline; Odia, Ikponmwonsa; Hass, Meike; Gabriel, Martin; Ölschläger, Stephan; Becker-Ziaja, Beate et al. (2012-09-27). "Molecular Diagnostics for Lassa Fever at Irrua Specialist Teaching Hospital, Nigeria: Lessons Learnt from Two Years of Laboratory Operation". PLoS Neglected Tropical Diseases 6 (9). doi:10.1371/journal.pntd.0001839. ISSN 1935-2727. PMID 23029594. PMC 3459880.

46. Olalekan, Adebimpe Wasiu (2016-11-02). "Pre-epidemic preparedness and the control of Lassa fever in Southern Nigeria". Research Journal of Health Sciences 4 (3): 243. doi:10.4314/rejhs.v4i3.7. ISSN 2467-8252.

47. "National Guidelines for Lassa fever case management" (2018), Nigeria Center for Disease Control

48. Raabe, Vanessa N.; Kann, Gerrit; Ribner, Bruce S.; Morales, Andres; Varkey, Jay B.; Mehta, Aneesh K.; Lyon, G. Marshall; Vanairsdale, Sharon et al. (2017-09-01). "Favipiravir and Ribavirin Treatment of Epidemiologically Linked Cases of Lassa Fever". Clinical infectious diseases: an official publication of the Infectious Diseases Society of America 65 (5): 855-859. doi:10.1093/cid/cix406. ISSN 1058-4838. PMID 29017278. PMC 5682919.
49. Tyring, Stephen K. (Stephen Keith) (2005). Antiviral agents, vaccines, and immunotherapies. New York: Marcel Dekker. ISBN 9780824754082. OCLC 58604581.

50. "Ribavirin". www.drugbank.ca. Retrieved 2019-05-25.

51. "Ribavirin" (in en). Wikipedia. 2019-06-05.

52. "Common Side Effects of Rebetol (Ribavirin) Drug Center". RxList. Retrieved 2019-05-25.

53. "Rebetol (Ribavirin): Side Effects, Interactions, Warning, Dosage \& Uses". RxList. Retrieved 2019-05-25.

54. "Rebetol, Ribasphere (ribavirin) dosing, indications, interactions, adverse effects, and more". reference.medscape.com. Retrieved 2019-05-25.

55. "Ribavirin for Lassa Fever Postexposure Prophylaxis" (PDF). wwwnc.cdc.gov. Retrieved 2017-04-16.

56. Hadi, Christiane M.; Goba, Augustine; Khan, Sheik Humarr; Bangura, James; Sankoh, Mbalu; Koroma, Saffa; Juana, Baindu; Bah, Alpha et al. (2010-12). "Ribavirin for Lassa Fever Postexposure Prophylaxis". Emerging Infectious Diseases 16 (12): 2009-2011. doi:10.3201/eid1612.100994. ISSN 1080-6040.

57. "Lassa fever - prognosis". bestpractice.bmj.com. BMJ. Retrieved 2019-06-27.

58. "Lassa Fever". www.sterlinghealthmcs.com. Retrieved 2019-0525.

59. I., Donaldson, Ross (2009). The Lassa ward: one man's fight against one of the world's deadliest diseases (1st ed ed.). New York: St. Martin's Press. ISBN 0312377002. OCLC 262885308. 